\title{
EFFECT OF T-2 MYCOTOXIN ON GLUT-2 EXPRESSION IN THE BROILER'S GASTROINTESTINAL TRACT
}

\author{
Piret Hussar ${ }^{1}$, Florina Popovska-Percinic ${ }^{2}$, \\ Lazo Pendovski ${ }^{2}$, Katerina Blagoevska ${ }^{2}$ \\ ${ }^{1}$ Faculty of Medicine, University of Tartu, Tartu, Estonia \\ ${ }^{2}$ Faculty of Veterinary Medicine, Ss.Cyril \& Methodius University in Skopje, \\ Skopje, Republic of Macedonia
}

\begin{abstract}
T-2 toxin is a trichothecene mycotoxin. It is a naturally occurring mold byproduct of Fusarium spp. fungus which is toxic to humans and animals. Deleterious effects of these feed contaminants in animals are well documented, ranging from growth impairment, decreased resistance to pathogens, hepatoand nephrotoxicity to death. As the initial interaction of mycotoxins is with the gut epithelium the epithelium is repeatedly exposed to these toxins at higher concentrations than other tissues. Several methods for the determination of T-2 toxin based on traditional chromatographic, immunoassay, or mass spectroscopy techniques have been described. As up to now there is lack of knowledges about the effect of T-2 mycotoxin on the expression of glucose transporters in the proximal part of the gastrointestinal tract (GIT), the aim of our investigation was to study the experssion of glucose transporter-2 (GLUT-2) in the epithelial cells of glandular stomach and duodenal mucosa comparatively in normal and T-2 toxicated broilers. Material from the two parts of GIT was collected from six 7-day-old normal female broilers and six 7-day-old female broilers with T-2 toxicosis. The specimen were fixed with $10 \%$ formalin, embedded into paraffin, slices $7 \mu \mathrm{m}$ thick were cut followed by immunohistochemical staining with polyclonal primary antibody Rabbit anti-GLUT-2 carried out according to the manufacturers quidelines (IHC kit, Abcam, UK). The results showed the moderate expression of GLUT-2 in epithelial cells both in the glandular as well as in the duodenal mucosa in the control group's chicken and the weaker expression of GLUT-2 after T-2 toxin administration indicating to the reduced glucose transport in the gastrointestinal system during T-2 mycotoxicosis.
\end{abstract}

Keywords: T-2 mycotoxin; GLUT-2; broilers; gastrointestinal tract 


\section{INTRODUCTION}

The Fusarium trichothecenes can be divided in four groups: A, B, C and D. The most toxic type A trichothecene is T-2 mycotoxin (T-2 toxin) [13]. Due to the great adaptability fungi are posing a serious risk to the animal feed industry. Recently, fungi have been designated as a greater threat to animal, plant and ecosystem health than the other taxonomic classes of pathogens [8]. The affected commodity may become contaminated with toxic secondary fungal metabolites, known as mycotoxins. For example, in the USA, the economic cost due to three mycotoxins is estimated to be USD 900 million per year [7]. As intestinal cells are the first cells to be exposed to mycotoxins, and often at higher concentrations than other tissues, the intestinal investigations have gained significant interest over the last decade, and some publications have demonstrated that mycotoxins are able to compromise several key functions of the gastrointestinal tract, including the decreased surface area available for nutrient absorption, the modulation of nutrient transporters, or the loss of barrier function $[4,9]$.

T-2 toxin causes acute and chronic toxicity and induces apoptosis in the immune system and fetal tissues. Consequently, there is a possibility of human consumption of the animal products contaminated with T-2 toxin and its metabolites [13]. The data from research studies show that mycotoxins can compromise several intestinal functions, such as digestion, absorption, permeability, defense, and result in lower productivity and poor health of animals. The research data also contain the information about structural changes in the avian intestine induced by T-2 toxicosis [16, 19].

According to the literature the majority of the ingested toxin is absorbed in the proximal part of the GIT $[1,6]$. The reduced glucose absorption in jejunum and its rate of appearance in venous plasma has been described during T-2 toxin administration [12]. As it is very important to elucidate how mycotoxins modulate the activity of transporters involved in nutrient digestion and uptake, and subsequently, the consequences on nutrient digestibility and on metabolizable energy, the aim of our study was to investigate the expression of glucose transporter-2 (GLUT-2) which facilitates diffusive transport of intracellular glucose into bloodstream in normal and T-2 toxicated broiler's epithelium of glandular stomach and duodenum. GLUT-2 was used since the hexoses transport in birds occurs predominantely by passive transport $[3,10,18]$ and recent works [11] propose that GLUT2 is also involved in the apical transport of hexoses (glucose, galactose, fructose). 


\section{MATERIAL AND METHODS}

The material from the glandular stomach and duodenum was collected from twelve 7-day-old female broilers (Gallus gallus domesticus) divided into control ( 6 birds) and T-2 toxin ( 6 birds) groups. For the T-2 toxin group the application of T-2 toxin was compulsive per os with syringe, in a dose of $0.250 \mathrm{mg} /$ day/bird, for three consecutive days, starting from the fourth day of the experiment. All the birds were given feed and water ad libitum. Sacrifice of chicks was 24 hours after the last toxin application, thereafter the material from stomach and duodenum was removed. Specimen were fixed with $10 \%$ buffered formalin, embedded into paraffin and slices $7 \mu \mathrm{m}$ thick were cut. Slices were deparaffinized with xylene and rehydrated in a graded series of ethanol. Endogenous peroxidase activity was blocked with $3 \% \mathrm{H}_{2} \mathrm{O}_{2}$ and the sections were stained using Immunohistochemistry kit (Abcam, UK) according to the manufacture guidelines. Polyclonal rabbit antibody GLUT-2 served as primary antibodies (Abcam, UK). The biotinylated secondary antibody and the streptavidin-conjugated peroxidase were used for the detection using DAB as chromogen. Nuclei were counterstained with Harris Hematoxylin. Negative controls did not contain primary antibodies.

\section{RESULTS}

In normal 7-day-old broilers (control group) the stomach's glandular and duodenal epithelium the expression of GLUT-2 was moderate. Duodenal enterocytes' nuclei as well as cytoplasm were stained by GLUT-2 (Figures1-2).

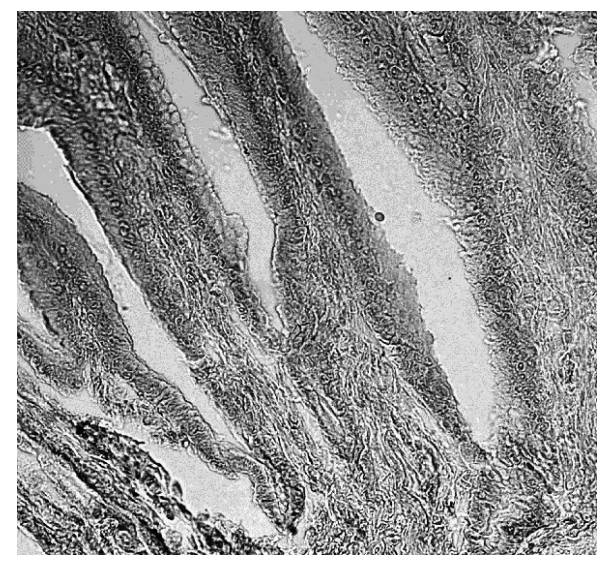

Figure 1. GLUT-2 moderately expressed in epithelial cells of 7-day-old broiler's glandular stomach (control group). 400x.

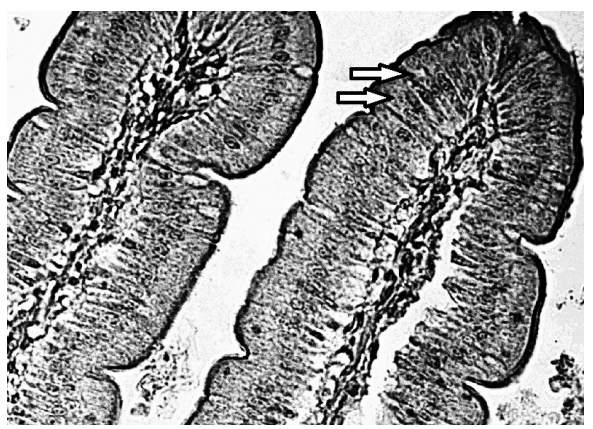

Figure 2. Expression of GLUT-2 in 7-dayold broiler's duodenal epithelium (control group). Note the stained cytoplasm (arrows) of enterocytes. $400 \times x$. 
In T-2 toxicated broiler's the expression of GLUT- 2 in epithelial cells in both investigated regions occurred to be weaker compared to the control group's chickens' epithelial cells. The intestinal enterocytes' cytoplasm remained weakly stained in all the toxicated birds (Figures 3-4).

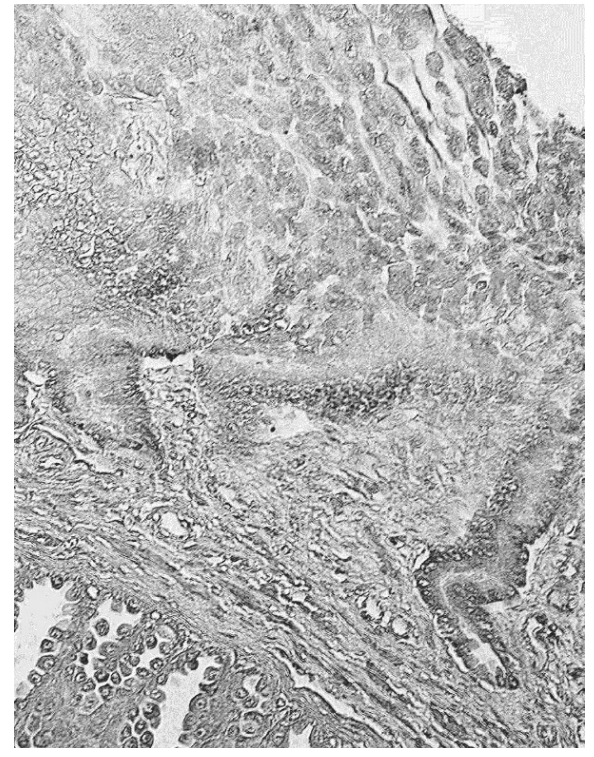

Figure 3. GLUT-2 weakly expressed in 7-day-old toxicated broiler's glandular stomach. 200x.

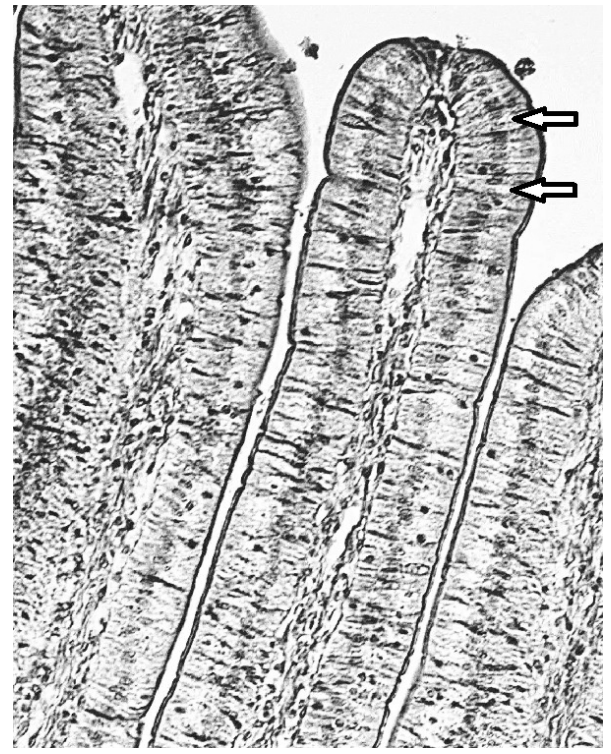

Figure 4. Expression of GLUT-2 in 7-day-old toxicated broiler's duodenal epithelium. The cytoplasm of enterocytes is weakly stained (arrows). 200x.

\section{DISCUSSION AND CONCLUSION}

Our investigations showed the weak expression of GLUT-2 in the epithelial cells of the glandular stomach as well as in duodenal mucosa in T-2 toxicated broilers compared to the control group where in both investigated regions the moderate expression of GLUT-2 was noted. The weak expression of GLUT-2 in the GIT's epithelial cells of T-2 toxicated birds indicates the reduced glucose transport during T-2 mycotoxicosis.

According to the literature different methods have been used to study the effects of T-2 on birds intestine [5,15]. The effect of T-2 toxin on glucose uptake has been studied by Suneja et al. with the results showing decrease in the glucose uptake after T-2 toxin oral administration [17]. The gene expression of SGLT1 and GLUT2 (facilitated glucose transporter) have been evaluated after Fusarium mycotoxin deoxynivalenol (DON) exposure [2]. The study revealed that the 
mRNA level of these genes was very low in the small intestine of chickens, suggesting that the down-regulation contributes to the inhibitory effect of (DON) on the intestinal glucose absorption. In our study GLUT-2, a transmembrane carrier protein that enables the passive movement of hexoses across cell membranes, was used. As the main apical transporter for active glucose uptake in small intestine is the sodium-dependent glucose cotransporter 1 (SGLT1), it could be used in our futher immunohistochemical investigations about the effects of T-2 mycotoxin.

The gastrointestinal system is exposed to all the mycotoxins in the contaminated feed which suggests that the intestinal epithelium is the major site for the effects of mycotoxin contaminated material. Therefore more studies in this field are required in future.

\section{REFERENCES}

1. Agence Française de Sécurité Sanitaire des Aliments (2009). Évaluation des risques liés à la présence de mycotoxines dans les chaînes alimentaires humaine et animale. Agence Française de Sécurité Sanitaire des Aliments, MaisonsAlfort. France.

2. Awad W.A., Vahjen W., Aschenbach J.R., Zentek J. (2011). A diet naturally contaminated with the Fusarium mycotoxin deoxynivalenol (DON) downregulates gene expression of glucose transporters in the intestine of broiler chickens. Livest Sci, 140, 72-79.

3. Barone S., Fussell S.L., Singh A.K. et al. (2009). S1c2a5 (Glut5) is essential for the absorption of fructose in the intestine and generation of fructose-induced hypertension. J Biol Chem, 284, 8, 5056-5066.

4. Blagoevska K., Dodovski A., Blagoevski A., Popovska-Percinik F., Pendovski L., Mickov L., Stojkovski V. (2012). Effect of Enterococcus faecium DSM 7134 probiotic upon some hematobiochemical values in acute ochratoxicoses in broilers. Mac Vet Rev, 35, 1, 43-49.

5. Blagoevska K., Popovska-Percinik F., Dodovski A., Radeski M., Blagoevski A., Mickov L., Jeremić D., Stojkovski V. (2014). The effect of the probiotic strain enterococcus faecium dsm 7134 on performances and protein status in experimental T-2 mycotoxicosis in broiler chicks. In: International VETistanbul Group Congress 2014, Istanbul, Turkey, abstract book, 2.

6. Cavret S., Lecoeur S. (2006). Fusariotoxin transfer in animal. Food Chem Toxicol, 44, 444-453.

7. Desjardins A., Maragos C., Norred W., Pestka J., Phillips T., Vardon P., Whitaker T., Wood G., van Egmond H. (2003). Mycotoxins, Risks in Plant,Animal,and Human System; Council for Agricultural Science and Technology: Ames, IA, USA. 
8. Fisher M.C., Henk, D.A., Briggs C.J., Brownstein J.S., Madoff L.C., McCraw S.L., Gurr S.J.(2012). Emerging fungal threats to animal, plant and ecosystem health. Nature 2012, 484, 186-194.

9. Grenier B., Applegate T.J. (2013). Modulation of Intestinal Functions Following Mycotoxin Ingestion: Meta-Analysis of Published Experiments in Animals. Toxins, 5, 2, 396-430.

10. Hussar P., Kärner M., Järveots T., Pendovski L., Duritis I., Popovska-Percinic F. (2016). Comparative study of glucose transporters GLUT-2 and GLUT-5 in ostriches gastrointestinal tract. Mac Vet Rev, 39,2, http://dx.doi.org/10.1515/macvetrev-2016-0089

11. Kellett G.L., Brot-Laroche E. (2005). Apical GLUT2: a major pathway of intestinal sugar absorption. Diabetes, 54, 10, 3056-3062.

12. Kumagai S., Shimizu T. (1988). Effects of Fusarenon-X and T-2 toxin on intestinal absorption of monosaccharide in rats. Arch Toxicol, 61, 489-495.

13. Li Y., Wang Z., Beier R.C., Shen J., De Smet D., De Saeger S., Zhang S.(2011). T-2 Toxin, a Trichothecene Mycotoxin: Review of Toxicity, Metabolism, and Analytical Methods. J Agric Food Chem, 59, 8, 3441-3453.

14. Osselaere A. (2013). Influence of deoxynivalenol and T-2 toxin on the intestinal barrier and liver function in broiler chickens. $\mathrm{PhD}$ dissertation, Ghent University.

15. Phletus P. Williams (1989). Effects of T-2 mycotoxin on gastrointestinal tissues: A Review of in vivo and in vitro models. Archives of Environmental Contamination and Toxicology 18, 3, 374-387.

16. Popovska-Percinic F., Blagoevska K., Dodovski A., Pendovski L., Petkov V., Blagoevska A., Stojkovski V., Dovenska M., Ristotski T. (2015). The effect of T-2 mycotoxicosis and probiotic administration on broiler's duodenal morphology. In International VETistanbul Group Congress 2015, Saint-Petersburg, Russia, abstract book, 705.

17. Suneja S.K., Ram G.C., Wagle D.S. (1984). Effects of T-2 toxin on glucose and tryptophan uptake and intestinal mucosal enzymes. Toxicon, 22, 1, 39-43.

18. Uldry M., Thorens B. (2004). The SLC2 family of facilitated hexose and polyol transporters. Pflügers Arch, 447, 480-489.

19. Witlock D.R., Wyatt R.D., Ruff M.D. (1977). Morphological changes in the avian intestine induced by citrinin and lack of effect of aflatoxin and T-2 toxin as seen with scanning electron microscopy. Toxicon, 15, 1, 41-44.

\section{Address for correspondence:}

Piret Hussar, M.D., D.M.Sc

Chair of Histology and Embryology, Department of Anatomy

Institute of Biomedicine and Translational Medicine, Faculty of Medicine

University of Tartu, Ravila 19, Tartu 50411, Estonia

E-mail: piret.hussar@ut.ee 